\title{
Constraints on cosmic-ray efficiency in the supernova remnant RCW 86 using multi-wavelength observations
}

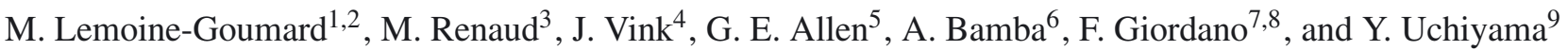 \\ 1 Université Bordeaux 1, CNRS/IN2P3, Centre d'Études Nucléaires de Bordeaux Gradignan, 33175 Gradignan, France \\ e-mail: lemoine@cenbg.in2p3.fr \\ 2 Funded by contract ERC-StG-259391 from the European Community \\ ${ }^{3}$ Laboratoire Univers et Particules de Montpellier, Université Montpellier 2, CNRS/IN2P3, 34095 Montpellier Cedex 5, France \\ e-mail: mrenaud@lupm. univ-montp2.fr \\ 4 Astronomical Institute, Utrecht University, PO Box 80000, 3508 TA Utrecht, The Netherlands \\ 5 MIT Kavli Institute for Astrophysics and Space Research, Cambridge, MA 02139, USA \\ ${ }^{6}$ Department of Physics and Mathematics, Aoyama Gakuin University 5-10-1 Fuchinobe, Chuo-ku, Sagamihara, \\ 252-5258 Kanagawa, Japan \\ 7 Dipartimento di Fisica "M. Merlin" dell’Universitá e del Politecnico di Bari, 70126 Bari, Italy \\ ${ }^{8}$ Istituto Nazionale di Fisica Nucleare, Sezione di Bari, 70126 Bari, Italy \\ 9 W. W. Hansen Experimental Physics Laboratory, Kavli Institute for Particle Astrophysics and Cosmology, \\ Department of Physics and SLAC National Accelerator Laboratory, Stanford University, Stanford, CA 94305, USA
}

Received 26 June 2012 / Accepted 19 July 2012

\begin{abstract}
Context. Several young supernova remnants (SNRs) have recently been detected in the high-energy (HE; $0.1<E<100 \mathrm{GeV}$ ) and very-high-energy (VHE; $E>100 \mathrm{GeV}$ ) gamma-ray domains. As exemplified by RX J1713.7-3946, the nature of this emission has been hotly debated, and direct evidence for the efficient acceleration of cosmic-ray protons at the SNR shocks still remains elusive. Aims. We study the broadband gamma-ray emission from one of these young SNRs, namely RCW 86, for which several observational lines of evidence indirectly point towards the presence of accelerated hadrons. We then attempt to detect any putative hadronic signal from this SNR in the available gamma-ray data, in order to assess the level of acceleration efficiency.

Methods. We analyzed more than 40 months of data acquired by the Large Area Telescope (LAT) on-board the Fermi Gamma-Ray Space Telescope in the HE domain, and gathered all of the relevant multi-wavelength (from radio to VHE gamma-rays) information about the broadband nonthermal emission from RCW 86. For this purpose, we re-analyzed the archival X-ray data from the ASCA/Gas Imaging Spectrometer (GIS), the XMM-Newton/EPIC-MOS, and the RXTE/Proportional Counter Array (PCA).

Results. Beyond the expected Galactic diffuse background, no significant gamma-ray emission in the direction of RCW 86 is detected in any of the $0.1-1,1-10$ and 10-100 GeV Fermi-LAT maps. The derived HE upper limits, together with the H.E.S.S. measurements in the VHE domain, are incompatible with a standard $E_{\mathrm{p}}^{-2}$ hadronic emission arising from proton-proton interactions, and can only be accommodated by a spectral index $\Gamma \leq 1.8$, i.e. a value in-between the standard (test-particle) index and the asymptotic limit of theoretical particle spectra in the case of strongly modified shocks. In such a hadronic scenario, the total energy in accelerated particles is at the level of $\eta_{\mathrm{CR}}=E_{\mathrm{CR}} / E_{\mathrm{SN}} \sim 0.07 d_{2.5}^{2} \mathrm{kpc} / \bar{n}_{\mathrm{cm}^{-3}}$ (with the distance $d_{2.5 \mathrm{kpc}} \equiv d / 2.5 \mathrm{kpc}$ and the effective density $\bar{n}_{\mathrm{cm}}{ }^{-3} \equiv$ $\bar{n} / 1 \mathrm{~cm}^{-3}$ ), and the average magnetic field must be stronger than $50 \mu \mathrm{G}$ in order to significantly suppress any leptonic contribution. On the other hand, the interpretation of the gamma-ray emission by inverse Compton scattering of high energy electrons reproduces the multi-wavelength data using a reasonable value for the average magnetic field of $15-25 \mu \mathrm{G}$. In this leptonic scenario, we derive a conservative upper limit to $\eta_{\mathrm{CR}}$ of $0.04 \mathrm{~d}_{2.5 \mathrm{kpc}}^{2} / \bar{n}_{\mathrm{cm}}{ }^{-3}$. We discuss these results in the light of existing estimates of the magnetic field strength, the effective density and the acceleration efficiency in RCW 86.
\end{abstract}

Key words. ISM: individual objects: RCW 86 - ISM: individual objects: G315.4-2.3 - ISM: individual objects: MSH 14-63 gamma rays: general - ISM: supernova remnants

\section{Introduction}

Supernova remnants (SNRs) are thought to be the primary sources of the bulk of Galactic cosmic-ray (CR) protons observed at Earth, up to the knee energy at $\sim 3 \mathrm{PeV}$. This paradigm mainly relies on the need to have sufficiently energetic sources that could provide the necessary power to maintain the Galactic CR energy density (e.g. Fields et al. 2001), and on the dominance of SNRs among sources of non-thermal radio emission. Our understanding of CR acceleration in SNRs mainly relies on the so-called Diffusive Shock Acceleration theory (in its non-linear version NLDSA, see Malkov \& Drury 2001), which is commonly invoked to explain several observational (though, indirect) lines of evidence for efficient particle acceleration at the SNR forward shocks up to very high energies. Among the requisites that must be fulfilled by this theory (see Blasi 2010, for a recent review), the observed broadband nonthermal emission of individual SNRs is of particular interest. This emission, arising from accelerated particles that emit photons through several channels (synchrotron $[\mathrm{SC}]$, inverseCompton [IC], nonthermal bremsstrahlung, proton-proton interactions and subsequent $\pi^{0}$ decay), offers new insights into 
the particle acceleration mechanisms at work in these sources, given the large number of multi-wavelength observations available nowadays towards many Galactic SNRs.

In particular, recent observations of young SNRs in the high-energy (HE; $0.1<E<100 \mathrm{GeV}$ ) and very-high-energy (VHE; $E>100 \mathrm{GeV}$ ) gamma-ray domains have raised several questions and triggered numerous theoretical investigations (e.g. Zirakashvili \& Aharonian 2010; Berezhko \& Völk 2010; Fang et al. 2011; Caprioli 2011). The critical issue regarding the nature of the observed gamma-ray emission has been intensively discussed in the literature in recent years (Ellison et al. 2010, and references therein). Nevertheless, these joint HE/VHE observations can help us to discriminate spectrally between the leptonic (through IC emission) and hadronic (through $\pi^{0} \rightarrow 2 \gamma$ ) scenarios, as shown by the Fermi-LAT (Abdo et al. 2011a) and H.E.S.S. (Aharonian et al. 2007) observations towards the TeV-bright SNR RX J1713.7-3946, which tend to support a leptonic model for the observed gamma-ray emission (Ellison et al. 2012; Li et al. 2011).

RCW 86 (Rodgers et al. 1960), also known as G315.4-2.3 or MSH 14-63 (Mills et al. 1961), is a $42^{\prime}$ diameter Galactic SNR in the southern sky. There has been much controversy about the nature of the supernova (SN) progenitor, and the SNR age and distance. This has resulted from the difficulties in reconciling the young age of RCW 86, based on its connection with the first historical SN ever recorded in AD 185, with its large size, given the relatively large distance estimates, which place RCW 86 at 2.3-2.8 kpc (Rosado et al. 1996; Sollerman et al. 2003) near an OB stellar association. Williams et al. (2011) recently reviewed all the arguments about the nature of the SN progenitor and critically examined the available observations of RCW 86. They suggest that the SNR is likely the remnant of a type Ia $\mathrm{SN}$, and, from hydrodynamic simulations, that the offcenter explosion occurred in a low-density cavity carved by the progenitor system (see also Vink et al. 1997). This scenario allows one to explain at once the young age and the large distance of RCW 86, which we scale in terms of $d_{2.5} \equiv d /(2.5 \mathrm{kpc})$ throughout the paper.

The general outline of its nearly circular shell is similar in the radio (Whiteoak \& Green 1996; Dickel et al. 2001), infrared (IR, Williams et al. 2011), optical (e.g. Smith 1997), and X-ray (Vink et al. 1997; Bamba et al. 2000; Bocchino et al. 2000; Borkowski et al. 2001b; Rho et al. 2002; Vink et al. 2006) domains, although significant fine-scale differences have been reported (e.g. Rho et al. 2002, and Fig. 2). X-ray observations towards RCW 86 have revealed the presence of both thermal and nonthermal emission, with very distinct morphologies: while soft X-rays correlate with optical emission from non-radiative shocks and IR emission from collisionally heated dust, the continuum hard X-ray emission is seen at the edges of radio emission. The high-temperature plasma, which mostly contains the strong $\mathrm{Fe} \mathrm{K}_{\alpha}$ line emission, also shows a particular morphology extending towards the SNR interior, as revealed with Suzaku, and is thought to originate from Fe-rich ejecta heated by the reverse shock (Ueno et al. 2007; Yamaguchi et al. 2008). The global distribution of the Fe-rich plasma in the entire SNR has recently been mapped with Suzaku (Yamaguchi et al. 2011), and the total Fe mass of $\sim 1 M_{\odot}$ deduced from these observations, together with a relatively low ejecta mass of $1-2 M_{\odot}$, strengthens the scenario of a type Ia SN at the origin of RCW 86.

Physical conditions (shock speed, ambient density, and magnetic field) vary greatly along the shell-like structure. In particular, slow shocks ( 600-800 $\mathrm{km} \mathrm{s}^{-1}$, see Long \& Blair 1990; Ghavamian et al. 2001) and relatively high post-shock densities
( $\sim 2 \mathrm{~cm}^{-3}$, see Williams et al. 2011$)$ have been measured in the southwest (SW) and northwest (NW) regions, while the northeast $(\mathrm{NE})$ region exhibits much faster shocks $\left(\gtrsim 2700 \mathrm{~km} \mathrm{~s}^{-1}\right.$ and $6000 \pm 2800 \mathrm{~km} \mathrm{~s}^{-1}$, see Vink et al. 2006; Helder et al. 2009) and lower densities $\left(\sim 0.1-0.3 \mathrm{~cm}^{-3}\right.$, e.g. Yamaguchi et al. 2008). In this region, Helder et al. (2009) argued that at least $50 \%$ of the total pressure is induced by CRs, based on the discrepancy between the measured shock velocity and the spectroscopically determined post-shock proton temperature (see also Vink et al. 2010). Moreover, Vink et al. (2006) found evidence for a concave electron spectrum, as predicted by the NLDSA theory, in order to explain the radio and X-ray SC emission observed from the same region. These two measurements, together with the recent detection of RCW 86 with the H.E.S.S. experiment in the VHE domain (Aharonian et al. 2009), seem to point towards an efficient CR source. However, complementary HE observations are needed to probe the nature of the gamma-ray emission, as discussed above.

We here report on Fermi-LAT observations and data analysis towards RCW 86 (Sect. 2). In an attempt to constrain the broadband nonthermal emission from the SNR, we present a reanalysis of the archival ASCA/GIS, XMM-Newton/EPIC-MOS, and RXTE/PCA X-ray data (Sect. 3), and collect the available information in the radio and VHE gamma-ray domains. We then discuss the constraints derived on the parent particle spectrum and on the subsequent acceleration efficiency, in the light of existing estimates (Sect. 4).

\section{Fermi-LAT observations and data analysis}

\subsection{Observations}

The LAT is a gamma-ray telescope that detects photons by conversion into electron-positron pairs in the energy range from $20 \mathrm{MeV}$ to more than $300 \mathrm{GeV}$, as described by Atwood et al. (2009). It is made of a high-resolution converter/tracker (for direction measurement of the incident gamma-rays), a $\mathrm{CsI}(\mathrm{Tl})$ crystal calorimeter (for energy measurement) and an anti-coincidence detector to reject the background of charged particles. The LAT has a large effective area $\left(\sim 8000 \mathrm{~cm}^{2}\right.$ on-axis above $1 \mathrm{GeV})$, a wide field of view $(\sim 2.4 \mathrm{sr})$ and good angular resolution $\left(\sim 0.6^{\circ}\right.$ radius for $68 \%$ containment at $1 \mathrm{GeV}$ for events converting in the front section of the tracker). The onorbit calibration is described in Abdo et al. (2009a).

The analysis used more than 40 months of data collected starting on August 4, 2008, and extending until February 22, 2012. We selected events with energies greater than $0.1 \mathrm{GeV}$, and excluded those with zenith angles larger than $100^{\circ}$, to minimize contamination from secondary gamma rays from the Earth's atmosphere (Abdo et al. 2009b). In addition, we excluded the time intervals when the rocking angle was more than $52^{\circ}$ and when the Fermi satellite was within the South Atlantic Anomaly. We used the P7SOURCE_V6 Instrument Response Functions (IRFs), and selected the "Source" events, which correspond to the best compromise between the number of selected photons and the charged particle residual background for the study of point-like or slightly extended sources.

\subsection{Analysis and results}

For the spectral analysis, a spectral-spatial model containing diffuse and point-like sources was created, and the parameters 


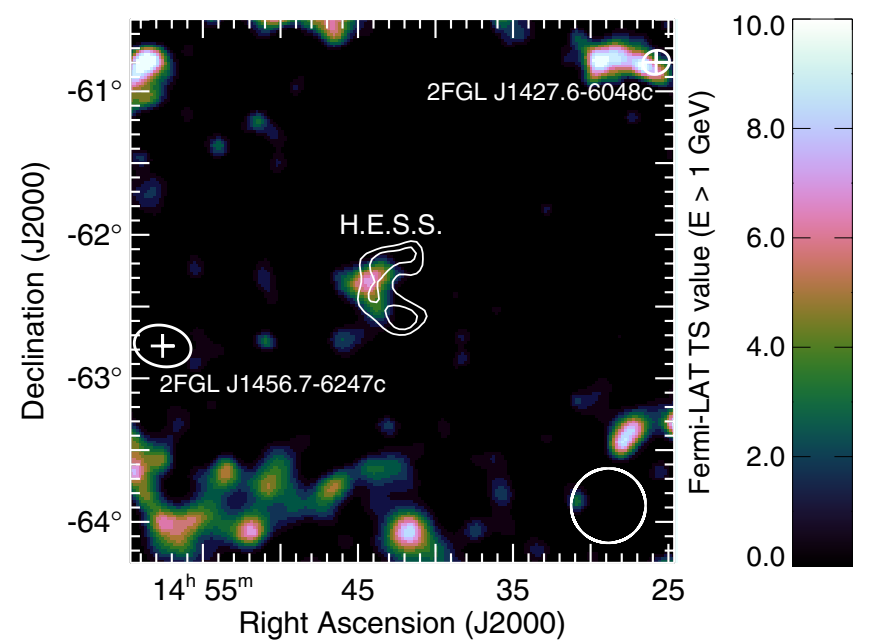

Fig. 1. Fermi-LAT test statistic (TS) map towards RCW 86, above $1 \mathrm{GeV}$. The TS was evaluated by placing a point-source at the center of each pixel, Galactic diffuse emission and nearby 2FGL sources being included in the background model. H.E.S.S. contours (Aharonian et al. 2009) are overlaid as white solid lines, and the white circle indicates the size of the $68 \%$ containment region of the LAT PSF at $10 \mathrm{GeV}$. Crosses and ellipses mark the best-fit positions and associated errors of the two sources from the 2FGL Fermi-LAT catalog (Nolan et al. 2012) within $4^{\circ}$ from RCW 86.

were obtained from a maximum likelihood fit to the data using the gtlike code implemented in the Fermi Science Tools ${ }^{1}$. The likelihood tool gtlike fits a source model to the data along with models for the residual charged particles and diffuse gamma-ray emission. For the Galactic diffuse emission, we used the ringhybrid model gal_2yearp7v6_v0.fits. The instrumental background and the extragalactic radiation are described by a single isotropic component with the spectral shape in the tabulated model iso p7v6source.txt. These models are available from the Fermi Science Support Center ${ }^{2}$. All sources within $15^{\circ}$ of RCW 86 were extracted from the Fermi-LAT 2FGL catalog (Nolan et al. 2012) and added to our spectral-spatial model of the region. The spectral parameters of sources closer than $3^{\circ}$ to RCW 86 were allowed to vary in the likelihood fit, while the parameters of all other sources were fixed at the 2FGL values.

Using this spectral-spatial model, we computed the LAT test statistic (TS) map of the region containing RCW 86, as shown in Fig. 1. The TS is defined as twice the difference between the log-likelihood $L_{1}$ obtained by fitting a source model plus the background model to the data, and the log-likelihood $L_{0}$ obtained by fitting the background model only, i.e. TS = $2\left(L_{1}-L_{0}\right)$. A TS $=25$ with two degrees of freedom (spectral index and normalization) roughly corresponds to a $4.6 \sigma$ detection significance. Figure 1 contains the TS value for a point source with fixed photon index $\Gamma=2$ at each map location, thus giving a measure of the statistical significance for the detection of a gamma-ray source in excess of the background. No significant excess suggesting gamma-ray emission is evident in the skymap, apart from faint excesses, which are unassociated with any 2FGL catalogued source, at the northern and southern borders of the map, and a faint hotspot located near the NE shell of RCW 86. Under the assumption of a pointlike source, this excess is found at $\mathrm{RA}(\mathrm{J} 2000) \approx 221.0^{\circ}$ and

\footnotetext{
1 http://fermi.gsfc.nasa.gov/ssc/data/

2 http://fermi.gsfc.nasa.gov/ssc/data/access/lat/

BackgroundModels.html
}

$\operatorname{Dec}(\mathrm{J} 2000) \approx-62.4^{\circ}$ with a TS value of 12 (i.e. $\sim 2.4 \sigma$ for four d.o.f., where the spectral index is allowed to vary). Therefore, we do not consider this hotspot in the following, bearing in mind that further Fermi-LAT observations in the coming years might provide confirmation of this indication of a signal towards RCW 86.

Since the Fermi-LAT 2FGL catalog does not contain any source in a region of $1^{\circ}$ around the SNR RCW 86, to determine an upper limit to the gamma-ray emission from RCW 86, we added an extended source consistent with the H.E.S.S. observations, i.e. a uniform disk centered at $\left(\mathrm{RA}(\mathrm{J} 2000)=220.68^{\circ}\right.$, $\left.\operatorname{Dec}(\mathrm{J} 2000)=-62.44^{\circ}\right)$ with a radius of $0.40^{\circ}$. We modeled the emission from RCW 86 using a power-law spectral distribution with two free parameters: the flux and the spectral index. The test statistic was employed to evaluate the significance of the gamma-ray fluxes coming from RCW 86 in three different energy bands: $0.1-1 \mathrm{GeV}, 1-10 \mathrm{GeV}$, and $10-100 \mathrm{GeV}$. In all tested energy bands, the likelihood ratio test indicate values of TS less than 25. This agrees with the non-detection in the 2FGL cata$\log$ as well as the LAT TS map presented in Fig. 1. Assuming a power-law shape for the source with a fixed spectral index of 2 , we then derived $99.9 \%$ confidence level (CL) upper limits to the flux in these three energy intervals using the Bayesian method proposed by Helene (1983). These upper limits, shown in Fig. 4, amount to $1.3 \times 10^{-8}, 1.1 \times 10^{-9}$, and $3.4 \times 10^{-10} \mathrm{ph} \mathrm{cm}^{-2} \mathrm{~s}^{-1}$, respectively. Varying the spectral index between 1.5 and 3 did not change significantly these estimates.

\section{X-ray observations}

We seek to estimate the total nonthermal X-ray emission from RCW 86 in order to constrain the underlying acceleration mechanisms, together with the gamma-ray observations presented above. There is a large amount of X-ray data towards the SNR, since ASCA, XMM-Newton, and more recently Suzaku have performed a nearly complete coverage (Bamba et al. 2000; Vink et al. 2006; Yamaguchi et al. 2011). In hard X-rays, the nonimaging instrument PCA onboard RXTE detected RCW 86 above $10 \mathrm{keV}$, and the original results were presented in Allen et al. (1999). From our point of view, it is worthwhile revisiting these archival X-ray data for several reasons. The RXTE/PCA data analysis presented below was carried out with the latest software release, and we investigate (and hence correct the extracted spectrum for) the contamination from the Galactic Ridge and the effect of the PCA off-axis response for such an extended source. For both ASCA/GIS and RXTE/PCA datasets, we also want to constrain the SC emission with the most advanced, physically-motivated, modeling dedicated to X-ray observations (Zirakashvili \& Aharonian 2010), rather than either the usual power-law approximation, which is obviously inadequate in the cutoff regime, or the often-used srcut model (Reynolds \& Keohane 1999), which relies on the SNR properties in the radio domain and hence makes the implicit assumption that radio and nonthermal X-rays arise from the same emission region, at odds with what is observed in RCW 86 (Rho et al. 2002, and Fig. 2).

\subsection{ASCA/GIS}

RCW 86 was observed with ASCA (Tanaka et al. 1994) on 1993 August 17, 18 with three pointings towards the NE, western (W), and SW regions. ASCA has two kinds of instruments, 


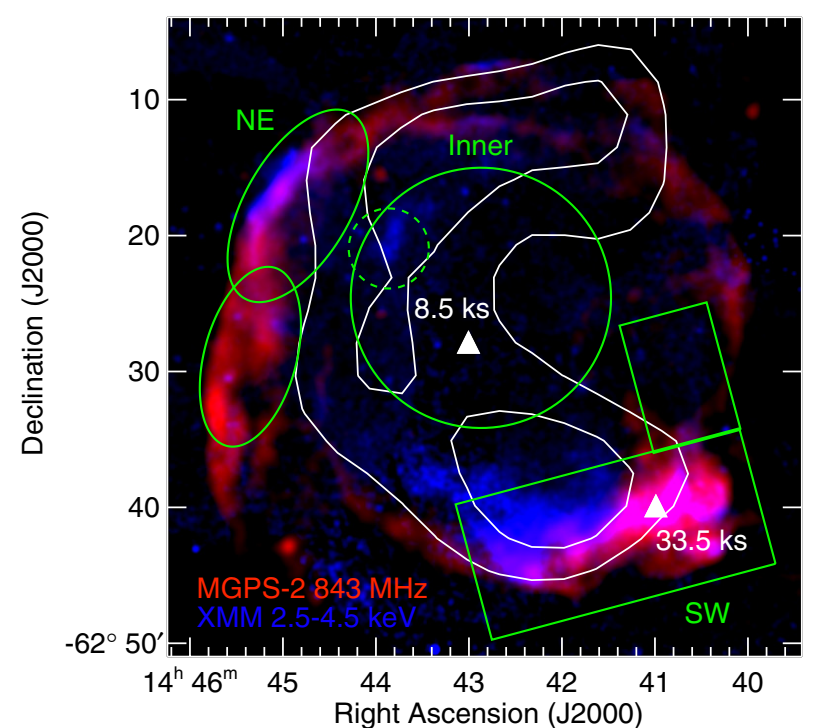

Fig. 2. Composite radio (MGPS-2 at $843 \mathrm{MHz}$, Murphy et al. 2007, in red) and X-ray (XMM-Newton/MOS in the 2.5-4.5 keV band, in blue) image of RCW 86. The XMM-Newton/MOS image has been smoothed with a Gaussian of width $10^{\prime \prime}$. H.E.S.S. contours (Aharonian et al. 2009) are overlaid in white, as in Fig. 1. Triangles mark the RXTE pointing positions with the associated exposure times. The different ASCA/GIS regions for spectral extraction are delineated in green and labeled NE, SW and Inner (counts within the dashed circle were excluded), as in Bamba et al. (2000).

GIS (Gas Imaging Spectrometers; Ohashi et al. 1996) and SIS (Solid-state Imaging Spectrometers; Burke et al. 1991), each at the focus of an X-ray Telescope (Serlemitsos et al. 1995). We focus on the GIS data set since it has a larger field of view and effective area. Data selection and analysis were performed following the procedure described in Bamba et al. (2000). The resulting exposure times are $\sim 20 \mathrm{ks}$ for NE, $\sim 9 \mathrm{ks}$ for $\mathrm{W}$, and $\sim 8 \mathrm{ks}$ for SW. We produced ASCA/GIS spectra from three distinct regions of RCW 86, which we labeled SW, NE and inner, as shown in Fig. 2.

Since RCW 86 exhibits both thermal and nonthermal $\mathrm{X}$-ray emission at different relative levels along the shell, we fit these spectra individually with an absorbed three-component model consisting of (1) a plane-parallel shock model with variable metal abundances (with their relative abundances fixed to solar ratios, pshock, Borkowski et al. 2001a), (2) an unresolved Gaussian line at fixed energy of $6.4 \mathrm{keV}$, and (3) the analytical approximation of the SC emission (Zirakashvili \& Aharonian 2010, ZA10 hereafter) from the shock-accelerated electron spectra derived by Zirakashvili \& Aharonian (2007) in the SC-losslimited regime. The first two components account for the lowtemperature thermal emission in non-equilibrium ionization and the Fe-K line detected in several regions along the SNR shell (Borkowski et al. 2001b; Ueno et al. 2007), respectively. We note that this iron line is most likely produced by a high-temperature ( $\gtrsim 5 \mathrm{keV}$ ) plasma (Bamba et al. 2000; Bocchino et al. 2000; Borkowski et al. 2001b; Rho et al. 2002), which we do not model here. However, we checked that adding such a component does not change significantly the best-fit results. The third component was used to describe the X-ray SC emission measured in RCW 86 (e.g. Borkowski et al. 2001b; Vink et al. 2006). We followed the ZA10 prescription, according to which the SC spectrum from shock-accelerated electrons in the downstream region (see Zirakashvili \& Aharonian 2007), in the case of Bohm

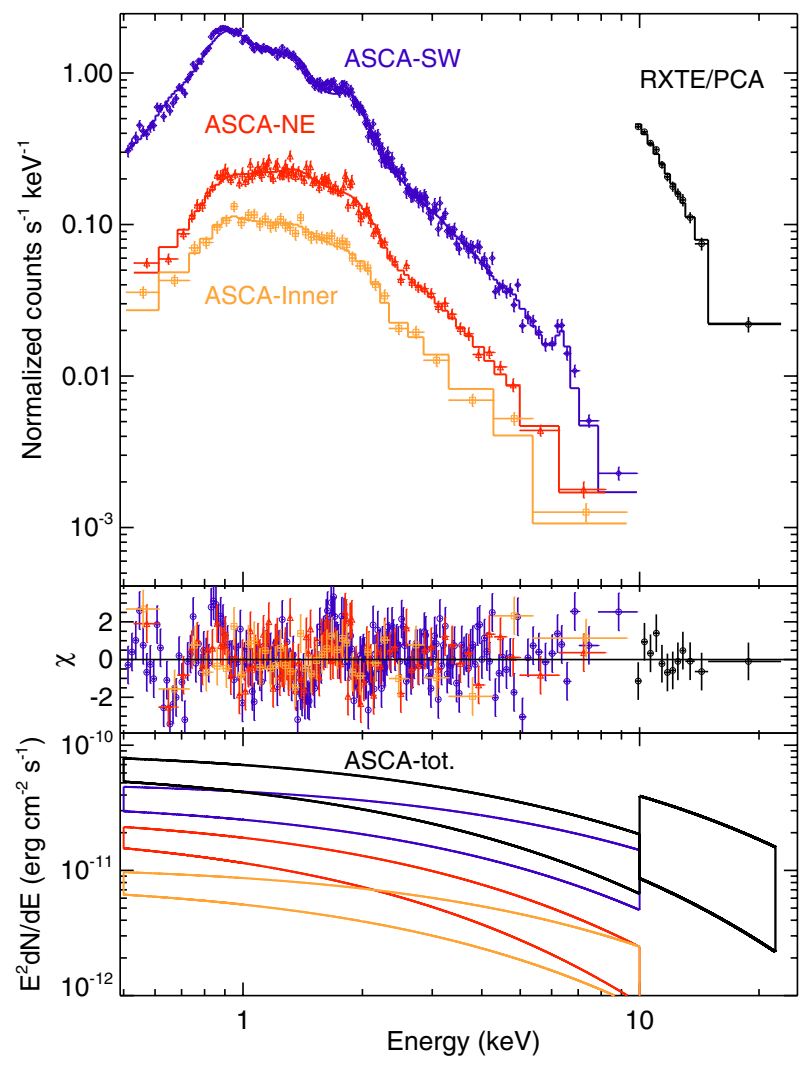

Fig. 3. $0.5-10 \mathrm{keV}$ ASCA/GIS spectra from the three regions considered in this work, together with the RXTE/PCA spectrum in the 10-25 keV energy range. All spectra have been rebinned to $10 \sigma$ per bin for sake of clarity. Solid lines represent the best-fit models (see text and Table 1). Residuals and unfolded nonthermal spectra are shown in the middle and lower panels, respectively.

diffusion and for a distributed (turbulent) magnetic field, is found to have the following behavior:

$$
\begin{aligned}
& \frac{\mathrm{d} N(E)}{\mathrm{d} E} \propto E^{-2}\left[1+0.185\left(\frac{E}{E_{1}}\right)^{0.4}\right]^{25 / 4.8} \times \exp \left[-\left(\frac{E}{E_{1}}\right)^{1 / 3}\right] \\
& E_{1}=\frac{0.056 \mathrm{keV}}{\eta_{B}}\left(\frac{V_{\mathrm{f}}}{3 \times 10^{3} \mathrm{~km} \mathrm{~s}^{-1}}\right)^{2}
\end{aligned}
$$

for which the upstream to downstream magnetic field ratio $\kappa$ is assumed to be $1 / \sqrt{11}, V_{\mathrm{f}}$ is the forward shock velocity, and $\eta_{B} \equiv$ $D / D_{\text {Bohm }} \geq 1$ is the factor allowing for deviation of the diffusion coefficient from its nominal value. The above expression is valid when the electron spectrum in the cutoff region is determined by the energy losses, i.e. when the SNR age is older than the SC cooling time of electrons $\tau \sim 1300 \eta_{B}^{1 / 2}\left(V_{\mathrm{f}} / 10^{3} \mathrm{~km} \mathrm{~s}^{-1}\right)^{-1}$ $(\mathrm{B} / 25 \mu \mathrm{G})^{-3 / 2}$ yr. Provided that the diffusion proceeds close to the nominal Bohm regime, this condition is met for RCW 86, which exhibits shock speeds in excess of $700 \mathrm{~km} \mathrm{~s}^{-1}$ and B-field estimates of at least $25 \mu \mathrm{G}$ (Völk et al. 2005; Vink et al. 2006).

The ASCA/GIS spectra and their respective best-fit models are shown in Fig. 3, while the spectral parameters are provided in Table 1. Results are in good agreement with those reported by Bamba et al. (2000) and Borkowski et al. (2001b), who both used ASCA data and different spectral models. Regarding the nonthermal emission, the best-fit values of $E_{1} \sim 0.01-0.02 \mathrm{keV}$ translate into shock speeds on the order of 1300-1800 $\sqrt{\eta_{B}} \mathrm{~km} \mathrm{~s}^{-1}$, which are roughly compatible with previous estimates for the different parts of the RCW 86 shell. 
Table 1. Spectral models for RCW 86.

\begin{tabular}{|c|c|c|c|c|}
\hline Model parameters & ASCA-SW & ASCA-NE & ASCA-Inner & RXTE/PCA \\
\hline$N_{\mathrm{H}}\left(\times 10^{21} \mathrm{~cm}^{-2}\right)$ & $1.78_{-0.44}^{+0.44}$ & $3.44_{-0.70}^{+0.98}$ & $0.10_{-0.10}^{+0.07}$ & - \\
\hline $\begin{array}{l}\text { pshock } \\
k \mathrm{~T}(\mathrm{keV}) \\
\tau\left(\times 10^{11} \mathrm{~cm}^{-3} \mathrm{~s}, \text { fixed }\right) \\
\text { Abundance } \\
\text { Norm }\left(\times 10^{-16} \int n_{\mathrm{e}} \mathrm{n}_{\mathrm{H}} \mathrm{dV} / 4 \pi \mathrm{d}^{2} \mathrm{~cm}^{-5}\right)\end{array}$ & $\begin{array}{c}0.74_{-0.07}^{+0.07} \\
0.7 \\
0.40_{-0.12}^{+0.16} \\
9.3_{-3.0}^{+2.7}\end{array}$ & $\begin{array}{c}0.42_{-0.06}^{+0.10} \\
1.5 \\
0.36_{-0.21}^{+0.81} \\
3.1_{-3.0}^{+9.9}\end{array}$ & $\begin{array}{c}1.70_{-1.05}^{+2.03} \\
2.0 \\
1 \text { (fixed) } \\
0.04_{-0.01}^{+0.32}\end{array}$ & $\begin{array}{l}- \\
- \\
- \\
-\end{array}$ \\
\hline $\begin{array}{l}\text { Gaussian } \\
\text { Line flux }\left(\times 10^{-5} \mathrm{~cm}^{-2} \mathrm{~s}^{-1}\right)\end{array}$ & $9.9_{-2.2}^{+2.2}$ & $2.7_{-1.2}^{+1.2}$ & $3.3_{-1.9}^{+1.9}$ & - \\
\hline $\begin{array}{l}\text { ZA10 synchrotron } \\
\text { Norm at } 1 \mathrm{keV}\left(\times 10^{-2} \mathrm{~cm}^{-2} \mathrm{~s}^{-1} \mathrm{keV}^{-1}\right) \\
E_{1}\left(\times 10^{-2} \mathrm{keV}\right)\end{array}$ & $\begin{array}{l}3.15_{-0.69}^{+0.40} \\
2.20_{-0.56}^{+0.94}\end{array}$ & $\begin{array}{l}1.76_{-0.30}^{+0.18} \\
1.01_{-0.21}^{+0.20}\end{array}$ & $\begin{array}{l}0.65_{-0.10}^{+0.11} \\
1.82_{-0.48}^{+0.67}\end{array}$ & $\begin{array}{l}6.18_{-1.70}^{+3.60} \\
2.29_{-0.68}^{+0.79}\end{array}$ \\
\hline$\chi^{2} / v$ & $575.8 / 416$ & $193.4 / 149$ & $176.8 / 168$ & $14.4 / 29$ \\
\hline
\end{tabular}

Notes. Uncertainties quoted here are provided at the $90 \%$ confidence level.

As shown in Table 1, the ionization ages $\tau$ from the pshock models are poorly constrained, and we fixed them to their nominal values while extracting the errors in the other free parameters. Finally, the three defined regions do actually not encompass the whole SNR. To estimate the nonthermal flux outside the ASCA regions, we used the $2.5-4.5 \mathrm{keV}$ XMM-Newton/ MOS image shown in Fig. 2, assuming that the flux in this band is entirely nonthermal. We found that $80 \%$ of the $2.5-4.5 \mathrm{keV}$ flux is contained within the ASCA regions, and we consider in the following the best-fit unfolded spectra shown in Fig. 3 (bottom) corrected by this factor.

\subsection{XMM-Newton/MOS}

XMM-Newton observed RCW 86 during several cycles, as part of the guaranteed time (GT, PI: Bleeker) program in 2000 and 2001 and as part of the guest observer program (PI: Vink) in 2004 and 2007. The advantage of XMM-Newton observations over the ASCA observations is that almost the entire SNR was covered, and that XMM-Newton has a larger effective area. This allowed us to estimate the total nonthermal X-ray flux, including that from regions of faint nonthermal emission outside the ASCA regions. Unfortunately, the very elliptical and high orbit of XMM-Newton results in a higher background rate than ASCA, which, moreover, is very irregular. To obtain a nonthermal flux estimate as accurately as possible, we limited ourselves to the observation IDs the least plagued by a high background signal. The data reduction was carried out with the standard XMM-Newton software SAS version 20110223. To limit the effects of chip-gaps and variable background, we limited the analysis to the EPIC-MOS instruments. Since the XMM-Newton observations were spread out over a large time interval, with many different, overlapping pointings, we based our nonthermal flux estimate on imaging rather than on a full spectral analysis, since for the latter one would need to take into account the different backgrounds per spectrum and different exposure weights for each single extraction region. The image for the EPIC-MOS 1 and 2 instruments, shown in Fig. 2, was made in the $2.5-4.5 \mathrm{keV}$ energy band. This band was used to estimate the total nonthermal flux, which starts dominating above $\sim 2 \mathrm{keV}$ although some S XV line emission can still be present at $2.45 \mathrm{keV}$. The cut-off at $4.5 \mathrm{keV}$ was used for practical reasons, as the relative background signal increases at higher energies. The effective area between 2.5 and $4.5 \mathrm{keV}$ is relatively flat and we adopt here the mean value of $345 \mathrm{~cm}^{2}$. The background estimates were based on the regions of the image outside RCW 86. This is not ideal, as the background rate varies from observation to observation, with an estimated background level of $(0.75-1.5) \times$ $10^{-4}$ events s$^{-1} \operatorname{arcsec}^{-2}$. The total count rate in the $2.5-4.5 \mathrm{keV}$ band for the region containing RCW 86 is 3.4 counts $\mathrm{s}^{-1}$ (for a total number of events of $3.7 \times 10^{5}$ ), which reduces to $1.2-2.3$ counts $\mathrm{s}^{-1}$ after background subtraction. To convert this count rate to nonthermal flux, we used two approaches. One was based on the analysis of the nonthermal emission from the NE region (Vink et al. 2006). For the MOS spectra of this region, we have both a flux estimate and a total $2.5-4.5 \mathrm{keV}$ count rate. Using the best-fit spectral index of $\Gamma=2.8$, we found that the conversion factor from count rate to nonthermal flux in the $2.5-4.5 \mathrm{keV}$ band is $\sim 1.7 \times 10^{-11} \mathrm{erg} \mathrm{cm}^{-2} \mathrm{~s}^{-1} /(\mathrm{cnt} / \mathrm{s})$. Hence, the $2.5-4.5 \mathrm{keV}$ nonthermal flux amounts to $(2.1-3.9) \times$ $10^{-11} \mathrm{erg} \mathrm{cm}^{-2} \mathrm{~s}^{-1}$, corresponding to a normalization at $1 \mathrm{keV}$ of (5.7-11) $\times 10^{-2} \mathrm{~cm}^{-2} \mathrm{~s}^{-1} \mathrm{keV}^{-1}$. One can also directly convert the net count rate to a flux rate using the effective area given above, which gives a normalization at $1 \mathrm{keV}$ of $(5.1-9.8) \times$ $10^{-2} \mathrm{~cm}^{-2} \mathrm{~s}^{-1} \mathrm{keV}^{-1}$. These values are consistent with the measurements by ASCA (and RXTE, see below and Table 1).

\subsection{RXTE/PCA}

The SNR was observed with RXTE for a total of $42 \mathrm{ks}$ between 1997 February 26 and 1997 March 17. The PCA (Jahoda et al. 2006) is comprised of an array of five coaligned proportional counter units (PCUs) that are mechanically collimated to have a field of view of $1^{\circ}$ (FWHM). For RCW 86, the on-axis effective area has a peak of about $4600 \mathrm{~cm}^{2}$ at $7.3 \mathrm{keV}$ and is greater than or equal to $10 \%$ of this value from about 2.4 to $27 \mathrm{keV}$. Between these energies, the fractional energy resolution $\Delta E / E$ ranges from about 0.25 to 0.12 (FWHM), respectively. The data for observation ID 20260 were downloaded from the HEASARC archive and reprocessed using version 6.11 of FTOOLS ${ }^{3}$. The tools xtefilt and maketime were used to produce a new filter file for each one of the five pointings in the observation (whose locations are shown in Fig. 2) and a set of good-time intervals (GTIs) for standard event selections, respectively. The GTIs, which include $38 \mathrm{ks}$ of data, and the tool saextrct were used to obtain a single, combined spectrum for the first layer of anodes

${ }^{3}$ http://heasarc.nasa.gov/ftools/ftools_menu.html 
in all five PCUs. An estimate of the combined spectrum of the instrumental and cosmic X-ray backgrounds was produced using the tool pcabackest with the latest combined faint-source model and SAA history file. As a test of the accuracy of this model, the total number of background counts in the last 50 of 129 pulse-height channels (i.e. at energies above $43 \mathrm{keV}$ ) is 698135 . For comparison, the total number of counts in the data in the same range is 697541 . The two values agree to better than $0.1 \%$ (i.e. to better than $1 \sigma$ ). A response file was produced using the tool pcarsp.

The spectral analysis was performed using the energy range from 10 to $25 \mathrm{keV}$. Some caution should be used when interpreting the results of the PCA spectral analysis because: (1) there are spatial variations in the cosmic X-ray background. In the 10-20 keV energy band, these variations are 0.2 counts s $\mathrm{s}^{-1}$ $(1 \sigma)$ for the top layer of anodes in all five PCUs ${ }^{4}$. For comparison, the background-subtracted emission from RCW 86 is 1.1 counts $\mathrm{s}^{-1}$ in this energy band. If an additional powerlaw component with a photon index of 1.3 (Ajello et al. 2008) is added to the fit to represent excess cosmic X-ray background emission, then the best fit normalization of this component is essentially zero and the $90 \%$ upper limit to the normalization corresponds to 0.3 counts $\mathrm{s}^{-1}$. (2) The background model does not include emission from the Galactic Ridge. Unfortunately, the analysis of the Galactic Ridge emission, as measured using the PCA (Valinia \& Marshall 1998), does not extend to the two pointing locations for RCW $86\left(\operatorname{RA}(J 2000)=220.75^{\circ}\right.$, $\operatorname{Dec}(\mathrm{J} 2000)=-62.46^{\circ}$ for the center and $\mathrm{RA}(\mathrm{J} 2000)=220.25^{\circ}$, $\operatorname{Dec}(\mathrm{J} 2000)=-62.67^{\circ}$ for the SW, see Fig. 2). However, there is a PCA observation (ID 30267) of a nearby, more or less sourcefree, region of the sky $\left(\mathrm{RA}(\mathrm{J} 2000)=214.55^{\circ}, \operatorname{Dec}(\mathrm{J} 2000)=\right.$ $\left.-63.93^{\circ}\right)$. An analysis of the data for this observation reveals little evidence of Galactic Ridge emission in the $10-25 \mathrm{keV}$ energy band, and we assume that we can ignore emission from the Galactic Ridge in our analysis. (3) While RCW 86 is entirely within the field of view of the telescope for each pointing, the SNR is not a point source and the relative PCA detection efficiency declines more or less linearly from one for an on-axis source to zero for a source that is $1^{\circ}$ off axis (Jahoda et al. 2006). Therefore, the amount of emission detected is less than the amount of emission that was produced by the SNR. On the basis of the XMM-Newton $2.5-4.5 \mathrm{keV}$ image and the RXTE pointing directions shown in Fig. 2, and the PCA off-axis response in the form $f=1-r / r_{0}$ with $r_{0}=0.965^{\circ}$ (Jahoda et al. 2006), we estimate this reduction factor to be $\sim 0.8$. The RXTE/PCA spectrum is shown in Fig. 3, and the best-fit parameters from the ZA10 prescription are provided in Table 1. Fitting the spectrum with a power-law gives acceptable and consistent results with those originally reported by Allen et al. (1999): $\Gamma=3.27 \pm$ 0.14 and a normalization at $1 \mathrm{keV}$ of $0.21_{-0.06}^{+0.08} \mathrm{~cm}^{-2} \mathrm{~s}^{-1} \mathrm{keV}^{-1}$ $\left(\chi^{2} /\right.$ d.o.f. $\left.=15 / 31\right)$. As for the total nonthermal ASCA spectrum, we consider in the following the best-fit unfolded PCA spectrum shown in Fig. 3 (bottom) corrected from this reduction factor.

\section{Discussion}

To constrain the nature of the gamma-ray emission from RCW 86, we have collected all the available information in the radio and VHE gamma-ray domains, in addition to the Fermi-LAT and X-ray data analyses presented in the previous sections. Observations towards RCW 86 have been carried out

\footnotetext{
4 http://universe.gsfc.nasa.gov/xrays/programs/rxte/ $\mathrm{pca} / \mathrm{doc} / \mathrm{bkg} / \mathrm{bkg}-2002$
}

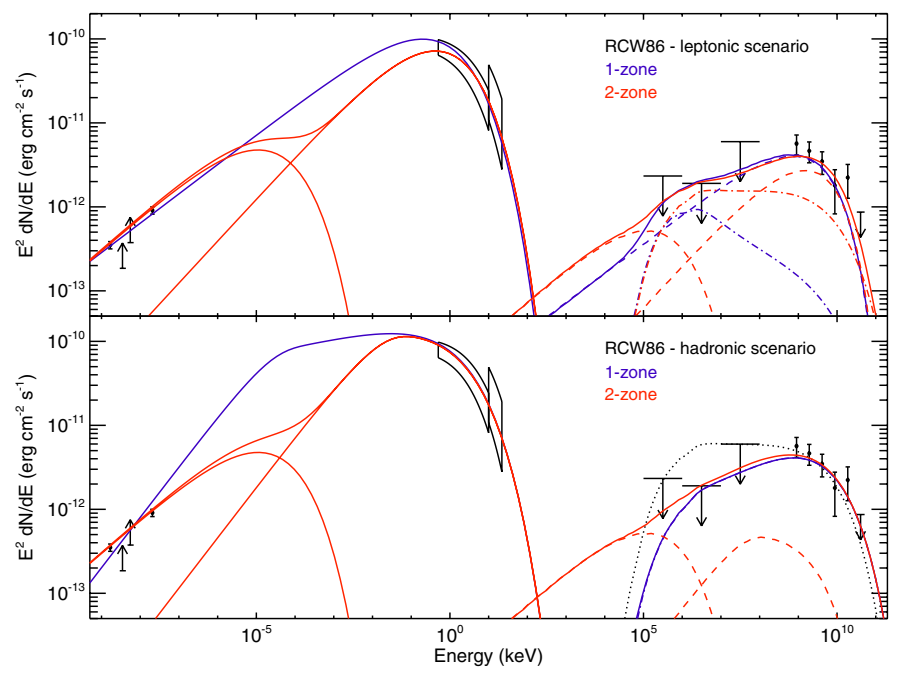

Fig. 4. Spectral energy distribution of RCW 86 with the best-fit leptonic (top) and hadronic (bottom) models. The radio data points are from Molonglo at $408 \mathrm{MHz}$ and Parkes at $5 \mathrm{GHz}$ (Caswell et al. 1975; Green 2011, priv. comm.), and lower limits from MOST at $843 \mathrm{MHz}$ and ATCA at $1.43 \mathrm{GHz}$ (Whiteoak \& Green 1996; Dickel et al. 2001). X-ray spectra from ASCA and RXTE were obtained as described in Sect. 3. The Fermi-LAT upper limits (99.9\% C.L.) derived in Sect. 2.2 and the H.E.S.S. data points in the VHE domain (Aharonian et al. 2009) are also shown. For each plot, the blue (respectively red) lines denote the total broadband emission from the one-zone (respectively two-zone) modeling discussed in Sect. 4, with SC, IC (with CMB only) and $\pi^{0}$ spectra shown as solid, dashed, and dash-dotted lines, respectively. The associated best-fit parameters are provided in Table 2. Note that in the bottom panel, $\pi^{0}$ emission is similar for the two models, and equals the total gamma-ray emission in the one-zone model (solid blue line). In addition, the dotted black line shows the (excluded) case of $\pi^{0}$ emission from a $E^{-2}$ hadron spectrum with $\eta_{\mathrm{p}} \sim 0.15 d_{2.5}^{2} / \bar{n}_{\mathrm{cm}^{-3}}$ and $E_{\max , \mathrm{p}}=100 \mathrm{TeV}$.

with several radiotelescopes. The flux densities measured with MOST at $408 \mathrm{MHz}$ (86 Jy) and Parkes at $5 \mathrm{GHz}(18.2 \mathrm{Jy})$ are extracted from Caswell et al. (1975), with respective errors of $10 \%$ (D. A. Green 2011, priv. comm.). The lower limits of 22 and $28 \mathrm{Jy}$ were derived at $843 \mathrm{MHz}$ (Whiteoak \& Green 1996) and $1.34 \mathrm{GHz}$ (Dickel et al. 2001), respectively. Spectral data points in the VHE domain were obtained with the H.E.S.S. experiment (Aharonian et al. 2009). The spectrum is well described with a power-law between 0.6 and $60 \mathrm{TeV}$, with a photon index of $2.54 \pm 0.12$ and a normalization at $1 \mathrm{TeV}$ of $(3.72 \pm 0.50) \times$ $10^{-12} \mathrm{~cm}^{-2} \mathrm{~s}^{-1} \mathrm{TeV}^{-1}$ (statistical errors only).

\subsection{Modeling the broadband emission of RCW 86}

Two mechanisms are commonly proposed to explain the gammaray emission in young SNRs: IC scattering off ambient photons (called leptonic scenario) and proton-proton interactions (called hadronic scenario). On the basis of a phenomenological approach, we aim to constrain the average magnetic field and the total energy in accelerated particles responsible for the broadband nonthermal spectrum of RCW 86 shown in Fig. 4. For this purpose, we modeled the $\pi^{0}$-decay gamma-ray emission from inelastic interactions of accelerated hadrons with ambient gas nuclei, according to the procedure described in Huang et al. (2007). The authors calculated the gamma-ray emission from the collisions between CR protons and helium nuclei and the interstellar medium with standard composition. We also computed the exact expressions of SC and IC emissions from a parent 
Table 2. Best-fit parameters of the leptonic and hadronic scenarios for the one-zone and two-zone models applied to the RCW 86 broadband emission (see Fig. 4).

\begin{tabular}{l|c|c|c|c|c|c}
\hline \hline & \multicolumn{3}{|c|}{ Leptonic model } & \multicolumn{3}{c}{ Hadronic model } \\
& One-zone & \multicolumn{2}{|c|}{ Two-zone } & One-zone & \multicolumn{2}{c}{ Two-zone } \\
& & radio & X-ray & & radio & X-ray \\
\hline$B(\mu \mathrm{G})$ & $15(25)$ & $>10(>25)$ & $15(25)$ & 400 & $>10(>25)$ & $>50$ \\
$\Gamma$ & 2.3 & 2.2 & 2.0 & 1.8 & 2.2 & 1.8 \\
$E_{\text {break,e }}(\mathrm{TeV})$ & - & - & - & 0.04 & - & 3.0 \\
$E_{\text {max,e }}(\mathrm{TeV})$ & $25(20)$ & $0.2(0.15)$ & $25(20)$ & 7 & $0.2(0.15)$ & 20 \\
$E_{\text {max,p }}(\mathrm{TeV})$ & 100 & - & 100 & 80 & - & 80 \\
$\eta_{\mathrm{e}}\left(\times 10^{-2} d_{2.5}^{2}\right)$ & $2(0.9)$ & $<2.5(<0.5)$ & $0.04(0.02)$ & 0.004 & $<2.5(<0.5)$ & 0.006 \\
$\eta_{\mathrm{p}}\left(\times 10^{-2} d_{2.5}^{2} / \bar{n}_{\mathrm{cm}^{-3}}\right)$ & $<2$ & - & $<4$ & 7 & - & 7 \\
$K_{\mathrm{ep}}$ & $>0.1(>0.05)$ & - & $>0.006(>0.003)$ & 0.002 & - & 0.001 \\
\hline
\end{tabular}

Notes. Numbers in parentheses correspond to the constraints obtained when considering the Galactic ISRFs from Porter \& Strong (2005) in the calculation of IC emission. Note that we do not consider any population of accelerated hadrons associated with the radio-SC-emitting electrons in the two-zone models ( $K_{\mathrm{ep}}$ is then left undetermined). We also fixed $E_{\mathrm{max}, \mathrm{p}}$ to $100 \mathrm{TeV}$ in the leptonic scenarios.

electron spectrum, as given in Blumenthal \& Gould (1970). Since the contribution to the IC scattering from the local interstellar (optical and infrared) radiation fields (ISRF), at the location of RCW 86, is unknown, we considered two cases: one with the cosmic microwave background (CMB) as the only source of seed photons for IC scattering, and the other with both the CMB and the Galactic ISRFs provided by Porter \& Strong (2005), which comprise the infrared (from dust, at $T \sim 35 \mathrm{~K}$ ) and optical (from stars, at $T \sim 4600 \mathrm{~K}$ ) emissions with respective energy densities of 0.66 and $0.94 \mathrm{eV} \mathrm{cm}^{-3}$. Nonthermal bremsstrahlung is neglected here given the relatively low densities $\left(\lesssim 1 \mathrm{~cm}^{-3}\right)$.

We qualitatively fit the free parameters of these leptonic and hadronic scenarios to the available data on RCW 86, and discuss the corresponding constraints on the parent particle spectrum, energetics, and magnetic field, in terms of the existing estimates. The particle spectra are assumed to follow a powerlaw with an exponential cutoff $\mathrm{d} N / \mathrm{d} E \propto E^{-\Gamma} \times \exp \left(-E / E_{\max }\right)$, with the same spectral index for both electrons and hadrons. We note that, in such a case, the resulting SC emission spectrum follows a power-law with an exponential cutoff in the form $\exp \left(-\left(E / E_{\text {cut }}\right)^{1 / 3}\right)$, which is similar to the ZA10 prescription that we used in Sect. 3 to fit the ASCA and RXTE spectra. We denote the fraction of the total energy injected into accelerated particles $\eta_{\mathrm{e}, \mathrm{p}}=W_{\mathrm{e}, \mathrm{p}} / E_{\mathrm{SN}}$, where $E_{\mathrm{SN}}$ is the energy of the SN explosion, assumed to be the canonical value of $10^{51} \mathrm{erg}^{5}$. We also calculate the so-called electron-to-proton ratio $K_{\mathrm{ep}}$ at momentum $1 \mathrm{GeV} \mathrm{c}^{-1}$, which is compared to the value measured in cosmic-rays $\left(K_{\text {ep }} \sim 10^{-2}\right)$. In these hadronic and leptonic scenarios, we consider two different models. The first is a one-zone model that assumes that radio, $\mathrm{X}$-rays, $\mathrm{GeV}$, and $\mathrm{TeV}$ gamma rays arise from the same emission region with a constant magnetic field. Since this model cannot account for the morphological difference between radio and X-rays (see Fig. 2), we also consider a two-zone model, which exhibits two populations of radio- and X-ray-/gamma-ray-emitting particles. The best-fit parameters for these two pairs of scenarios are given in Table 2.

\subsection{Leptonic scenario}

\subsubsection{One-zone model}

We assume that SC and IC photons are produced by highenergy electrons confined in the same emitting region. Figure 4

\footnotetext{
5 This assumption would be fairly valid in the case of RCW 86 is indeed the remnant of a type Ia SN (Williams et al. 2011; Yamaguchi et al. 2011).
}

(top, blue lines) shows that the broadband emission of RCW 86 can be easily accomodated using an injection spectrum with spectral index $\Gamma=2.3$, in agreement with the radio measurements, an exponential cutoff at $20-25 \mathrm{TeV}$, and an average magnetic field of $15-25 \mu \mathrm{G}$, depending on the level of ISRFs as seed photons in the calculation of IC emission. This one-zone model also provides an upper limit to the energy injected into accelerated hadrons of $\sim 2 \times 10^{49} d_{2.5}^{2} / \bar{n}_{\mathrm{cm}^{-3}} \mathrm{erg}$, with $\bar{n}$ the effective density $^{6}$, as depicted by the dash-dotted blue line in Fig. 4 (top). This estimate is only slightly sensitive to the assumed $E_{\text {max,p }}$, which we conservatively fixed here to $100 \mathrm{TeV}$. The resulting electron-to-proton ratio $K_{\text {ep }}$ amounts to $\gtrsim 0.05-0.1$.

\subsubsection{Two-zone model}

The above estimates rely on the implicit assumption that radio, X-ray and gamma-ray emission regions occupy the same volume. As shown by Rho et al. (2002) and visible in Fig. 2, this does not seem to be the case for RCW 86. Therefore, we consider two different populations of radio- and X-ray/gammaray-emitting leptons to reproduce the broadband emission of RCW 86. In this scenario, we use a harder lepton spectrum to explain the X-ray and VHE observations, with $\Gamma=2$, and a separate electron population to account for the radio emission, with $\Gamma=2.2$. For simplicity, we do not model any putative population of accelerated hadrons associated with the latter leptonic population, given the poor constraints obtained (see below). Figure 4 (top, red lines) shows a reasonable fit of the X-ray and gamma-ray data assuming an exponential cutoff at $20-25 \mathrm{TeV}$, and an average magnetic field of $15-25 \mu \mathrm{G}$, which is similar to that of the one-zone model. The direct consequence of this two-zone model is that it slightly relaxes the constraint on the energy injected into accelerated particles, owing to a harder spectrum. In this case, the estimated maximal energy injected into accelerated hadrons is $\sim 4 \times 10^{49} d_{2.5}^{2} / \bar{n}_{\mathrm{cm}^{-3}} \mathrm{erg}$, i.e. twice as large as in the one-zone model, and the resulting $K_{\text {ep }} \gtrsim(3-6) \times 10^{-3}$ gets closer to the observed value in CRs. Recently, Williams et al. (2011) performed a combined IR/X-ray

\footnotetext{
${ }^{6}$ For Bohm diffusion and a compression ratio of $4, \kappa=B_{\text {up }} / B_{\text {down }} \equiv$ $D_{\text {down }} / D_{\text {up }}=1 / \sqrt{11}$, as defined in Eq. (2), with $D_{\text {down }}$ (resp. $D_{\text {up }}$ ) the diffusion coefficient in the downstream (resp. upstream) medium. Given that the residence time of particles $t$ scales as $1 / D, \bar{n}=\left(n_{\text {up }} \times t_{\text {up }}+\right.$ $\left.n_{\text {down }} \times t_{\text {down }}\right) /\left(t_{\text {up }}+t_{\text {down }}\right) \sim 0.8 n_{\text {down }}$. Throughout the discussion, we then assume that $\bar{n}$ equals the post-shock density.
} 
analysis and derived post-shock densities in the NW and SW regions of the SNR of $2.0 \mathrm{~cm}^{-3}$ and $2.4 \mathrm{~cm}^{-3}$. If applicable to the whole SNR, these values would imply a maximal energy injected into hadrons of $\sim 2 \times 10^{49} d_{2.5}^{2} \mathrm{erg}$, much lower than what Helder et al. (2009) estimated in the NE region, where they evaluated that $>50 \%$ of the post-shock pressure is produced by CRs (see below, Sect. 5). However, the effective density in this region is known to be much lower than the western part of the shell. For instance, Yamaguchi et al. (2008) derived a post-shock density from the (weak) thermal X-ray emission of $0.26 f^{-0.5} \mathrm{~cm}^{-3}$ ( $f$ is the filling factor of this thermal component), and Helder et al. (2009) assumed a density of $0.1 \mathrm{~cm}^{-3}$, as derived in Vink et al. (2006). As for the radio-SC-emitting electrons, we found that the magnetic field in this zone must be stronger than $10-25 \mu \mathrm{G}$ (depending on the level of Galactic ISRFs). This estimate agrees with the value deduced by Vink et al. (2006) from the width of the filament in the NE region $(24 \pm 5 \mu \mathrm{G})$, but barely agrees with the one obtained by Bamba et al. (2005) for the SW filament ( 4-12 $\mu \mathrm{G})$. Völk et al. (2005) estimated a stronger magnetic field strength $\left(99_{-26}^{+46} \mu \mathrm{G}\right)$ from the thickness of the same SW filamentary structure observed by Rho et al. (2002). However, as noted by these authors, this estimate could be reduced by a factor of $\sim 2$, due to the non-spherical geometry, and hence the incomplete de-projection, of this feature, which lies in the interior of the remnant. We also note that Arbutina et al. (2012) found a volume-averaged magnetic field in RCW 86 of $\sim 70 \mu \mathrm{G}$, based on modified equipartition calculations applied to shell-type SNRs.

\subsection{Hadronic scenario}

\subsubsection{One-zone model}

In the hadronic scenario, gamma rays are predominantly radiated through decays of $\pi^{0}$ mesons produced in collisions between accelerated protons/nuclei and ambient gas. This directly implies that the average magnetic field needs to be much stronger than the previous estimates to suppress the IC component. In a first step, as performed above, we assume that high-energy electrons and protons are confined in the same emitting region. The standard $E^{-2}$-like spectrum with a fraction of total energy into hadrons of $\eta_{\mathrm{p}} \sim 0.15 d_{2.5}^{2} / \bar{n}_{\mathrm{cm}^{-3}}$, which would accommodate the H.E.S.S. measurements (as discussed in Aharonian et al. 2009), is clearly ruled out, as shown in Fig. 4 (bottom, dotted black line). The hard spectral index required is in-between the standard (test-particle) index and the asymptotic limit of $\Gamma=1.5$ predicted by extremely efficient CR acceleration (Malkov 1999; Berezhko \& Ellison 1999). This is similar to RX J1713.7-3946 (Abdo et al. 2011a) and, to a lesser extent, to RX J0852.0-4622 (aka Vela Junior, Tanaka et al. 2011). Fang et al. (2011) modeled the broadband emission of RCW 86 based on a semi-analytical solution to the NLDSA theory at SNR shocks. Their predictions are in conflict with the Fermi-LAT upper limits. It should be noted that the SC spectrum from electrons with the same in$\operatorname{dex} \Gamma=1.8$ is inconsistent with the radio spectral index deduced from the two flux points in Fig. 4. To reproduce the radio data from MOST and Parkes, the magnetic field should be high enough to produce significant energy losses during the lifetime of the remnant ( $\sim 1820 \mathrm{y})$ and create a break in the SC spectrum. A reasonable fit is obtained by using a total energy injected into accelerated hadrons of $\sim 7 \times 10^{49} d_{2.5}^{2} / \bar{n}_{\mathrm{cm}^{-3}}$ erg and an average magnetic field of $400 \mu \mathrm{G}$, with a break in the electron spectrum at $\sim 40 \mathrm{GeV}$. This magnetic field is much stronger than any estimates published so far on RCW 86, as discussed in Sect. 4.2, and $K_{\text {ep }} \sim 2 \times 10^{-3}$ is much lower than that measured in the CR spectrum.

\subsubsection{Two-zone model}

The constraint on the magnetic field is clearly relaxed in a twozone model in which the radio emission is produced by a separate electron population. In this case, a reasonable fit is shown in Fig. 4 (bottom, red lines). It assumes a (lower limit on the) magnetic field of $50 \mu \mathrm{G}$ in the X-ray-emitting zone (to suppress the IC component), with an injection spectrum with a power-law index of 1.8, an energy break at $3 \mathrm{TeV}$ (produced by SC cooling), and an exponential cut-off at $20 \mathrm{TeV}$. This two-zone model does not modify the estimated energy injected into accelerated hadrons, and the spectrum of radio-emitting electrons is also unchanged with respect to the two-zone leptonic model (Sect. 4.2.2). The resulting $K_{\mathrm{ep}}$ is still much lower than $10^{-2}$. In regard to the spectral shape deduced from these hadronic scenarios, we note that Vink et al. (2006) found that a concave lepton spectrum is favored over a simple power-law to explain the broadband SC emission from the NE region, even though they could not distinguish a flattening at high energies $\Gamma=2.2 \rightarrow 2$ from a flattening $\Gamma=2.2 \rightarrow 1.5$. The resulting $\pi^{0}$-decay emission from such a concave hadron spectrum (given that relativistic lepton and hadron indices should be very similar) would certainly mimic an $E^{-1.8}$ spectrum at the highest energies, although it is unclear whether the lower-energy part of such a spectrum would still agree with the Fermi-LAT upper limits. It is also interesting to note that, in a hadronic scenario, the gamma-ray emission is expected to be approximately proportional to the local density. Since the NW and SW regions both exhibit higher densities than the NE region, these regions should be brighter in the $\mathrm{TeV}$ regime, assuming a uniform $\mathrm{CR}$ density along the SNR shell. However, while the H.E.S.S. image looks similar to that observed in radio and X-rays, the azimuthal profile shown in Aharonian et al. (2009) is well-fit with a constant, without any clear variation along the shell. Deeper VHE observations are required to investigate in more detail the gamma-ray morphology of RCW 86 and help discriminate the origin of the gamma-ray emission.

\section{Conclusion}

The Fermi-LAT upper limits in the HE domain derived in this work for the young SNR RCW 86 provide strong constraints on the injection spectrum of the primary population responsible for the extended VHE emission. A hadronic scenario can only reproduce the multi-wavelength data using a hard proton spectrum (spectral index $\Gamma \leq 1.8$ ) and a total energy injected into hadrons residing in RCW 86 of $\sim 7 \times 10^{49}\left(\bar{n} / 1 \mathrm{~cm}^{-3}\right)^{-1} d_{2.5}^{2} \mathrm{erg}$. Given that the one-zone hadronic model suffers from several limitations (incompatible radio spectral index, very low $K_{\mathrm{ep}}$ and extremely high B-field), only a two-zone model can fulfill all the observational constraints, though still with a low $K_{\text {ep }}$. In the one- and two-zone leptonic scenarios, the multi-wavelength data can be closely reproduced using electron spectral indices of $\sim 2.0-2.3$, a total energy injected in electrons of $\sim 2 \times 10^{49} \mathrm{erg}$, and a reasonable average magnetic field of $15-25 \mu \mathrm{G}$. In such a case, the most conservative upper limit to the total energy injected into hadrons inside RCW 86 amounts to $\sim 4 \times 10^{49}\left(\bar{n} / 1 \mathrm{~cm}^{-3}\right)^{-1} d_{2.5}^{2} \mathrm{erg}$.

These estimates of the total CR energy content in RCW 86 can be translated into CR pressure $P_{\text {tot,CR }}=E_{\text {tot,CR }} / 3 \bar{V}$, where $\bar{V}$ is the effective CR volume within the SNR. Assuming that this volume is given by the best-fit parameters of the shell 
morphology observed with H.E.S.S. (though not statistically significant over a uniform sphere, see Aharonian et al. 2009), we obtain $\bar{V}=(6 \pm 2) \times 10^{59} d_{2.5}^{3} \mathrm{~cm}^{3}$, and $P_{\text {tot,CR }}$ must be conservatively lower than $3.8_{-1.0}^{+2.0} \times 10^{-10}\left(E_{\text {tot,p }} / 7 \times 10^{49}\right.$ erg $)$

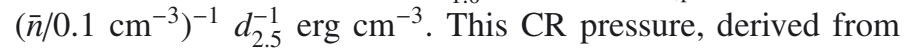
the modeling of the broadband nonthermal emission of the entire SNR (see Sect. 4), is very close to the CR pressure found by Helder et al. (2009) in the NE region, and later confirmed by Vink et al. (2010), $P_{\mathrm{NE}, \mathrm{CR}} \gtrsim 3.7 \times 10^{-10}\left(\bar{n} / 0.1 \mathrm{~cm}^{-3}\right)$

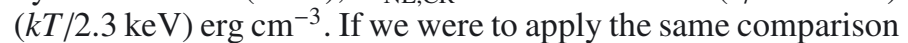
for the other regions along the RCW 86 shell, which all exhibit higher plasma densities $\left(0.5-2 \mathrm{~cm}^{-3}\right.$, see Yamaguchi et al. 2011; Williams et al. 2011), our upper limit to $P_{\text {tot,CR }}$ would most likely be inconsistent with the Helder et al. (2009) estimate regarding the fractional CR pressure. However, the large uncertainties in the effective CR volume in the above calculations, among others, prevent us from drawing firm conclusions about the acceleration efficiency in RCW 86.

Acknowledgements. The Fermi LAT Collaboration acknowledges generous ongoing support from a number of agencies and institutes that have supported both the development and the operation of the LAT as well as scientific data analysis. These include the National Aeronautics and Space Administration and the Department of Energy in the United States, the Commissariat à l'Energie Atomique and the Centre National de la Recherche Scientifique/Institut National de Physique Nucléaire et de Physique des Particules in France, the Agenzia Spaziale Italiana, the Istituto Nazionale di Fisica Nucleare, and the Istituto Nazionale di Astrofisica in Italy, the Ministry of Education, Culture, Sports, Science and Technology (MEXT), High Energy Accelerator Research Organization (KEK) and Japan Aerospace Exploration Agency (JAXA) in Japan, and the K. A. Wallenberg Foundation and the Swedish National Space Board in Sweden. Additional support for science analysis during the operations phase from the following agencies is also gratefully acknowledged: the Instituto Nazionale di Astrofisica in Italy and the Centre National d'Etudes Spatiales in France. We thank D. A. Green for helpful advice regarding radio measurements, and A. Marcowith for stimulating discussions about the modeling of the RCW 86 broadband emission.

\section{References}

Abdo, A. A., Ackermann, M., Atwood, W. B., et al. 2009a, ApJ, 696, 1084 Abdo, A. A., Ackermann, M., Ajello, M., et al. 2009b, Phys. Rev. D, 80, 12004 Abdo, A. A., Ackermann, M., Ajello, M., et al. 2011, ApJ, 734, 28

Aharonian, F. A., Akhperjanian, A. G., Bazer-Bachi, A. R., et al. 2007, A\&A, 464,235

Aharonian, F. A., Akhperjanian, A. G., de Almeida, U. B., et al. 2009, ApJ, 692, 1500

Ajello, M., Greiner, J., Sato, G., et al. 2008, ApJ, 689, 666

Allen, G. E. 1999, Proc. 26th ICRC, 3, 480

Arbutina, B., Urošević, D., Andjelić, M. M., et al. 2012, ApJ, 746, 79

Atwood, W. B., Abdo, A. A., Ackermann, M., et al. 2009, ApJ, 697, 1071

Bamba, A., Koyama, K., \& Tomida, H. 2000, PASJ, 52, 1157
Bamba, A., Yamazaki, R., Yoshida, T., et al. 2005, ApJ, 621, 793 Berezhko, E. G., \& Ellison, D. C. 1999, ApJ, 526, 385

Berezhko, E. G., \& Völk, H. J. 2010, A\&A, 511, 34

Blasi, P. 2010, Proc. ICATPP, Villa Olmo, in press [arXiv: 1012 . 5005]

Blumenthal, G. R., \& Gould, R. J. 1970, Rev. Mod. Phys., 42, 237

Bocchino, F., Vink, J., Favata, F., et al. 2000, A\&A, 360, 671

Borkowski, K. J., Lyerly, W. J., \& Reynolds, S. P. 2001a, ApJ, 548, 820

Borkowski, K. J., Rho, J., Reynolds, S. P., \& Dyer, K. K. 2001b, ApJ, 550, 334

Burke, B. E., Mountain R. W., Harrison, D. C., et al. 1991, ITED, 38, 1069

Caprioli, D. 2011, JCAP, 05, 026

Caswell, J. L., Clark, D. H., \& Crawford, D. F. 1975, AuJPA, 37, 39

Dickel, J. R., Strom, R. G., \& Milne, D. K. 2001, ApJ, 546, 447

Ellison, D. C., Patnaude, D. J., Slane, P., \& Raymond, J. 2011, ApJ, 712, 287

Ellison, D. C., Slane, P., Patnaude, D. J., \& Bykov, A. M. 2012, ApJ, 744, 39

Fang, J., Tang, Y., \& Zhang, L. 2011, ApJ, 731, 32

Fields, B. D., Olive, K. A., Cassé, M., \& Vangioni-Flam, E. 2001, A\&A, 370, 623

Ghavamian, P., Raymond, J., Smith, R. C., Hartigan, P., et al. 2001, ApJ, 547, 995

Helder, E. A., Vink, J., Bassa, C. G., et al. 2009, Science, 325, 719

Helene, O. 1983, Nucl. Instr. Meth. Phys. Res., 212, 319

Huang, C.-Y., Park, S.-E., Pohl, M., \& Daniels, C. D. 2007, APh, 27, 429

Jahoda, K., Markwardt, C. B., Radeva, Y., et al. 2006, ApJS, 163, 401

Li, H., Liu, S., \& Chen, Y. 2011, ApJ, 742, L10

Long, K. S., \& Blair, W. P. 1990, ApJ, 358, L13

Malkov, M. A. 1999, ApJ, 511, L53

Malkov, M. A., \& O’C Drury, L. 2001, Rep. Prog. Phys., 64, 429

Mills, B. Y., Slee, O. B., \& Hill, E. R. 1961, AuJPh, 14, 497

Murphy, T., Mauch, T., Green, A., et al. 2007, MNRAS, 382, 382

Nolan, P. L., Abdo, A. A., Ackermann, M., et al. 2012, ApJS, 199, 31 (2FGL catalog)

Ohashi, T., Ebisawa, K., Fukazawa, Y., et al. 1996, PASJ, 48, 157

Porter, T. A., \& Strong, A. W. 2005, Proc. 29th ICRC, 4, 77

Reynolds, S. P., \& Keohane, J. W. 1999, ApJ, 525, 368

Rho, J., Dyer, K. K., Borkowski, K. J., et al. 2002, ApJ, 581, 1116

Rodgers, A. W., Campbell, C. T., \& Whiteoak, J. B. 1960, MNRAS, 121, 103

Rosado, M., Ambrocio-Cruz, P., Le Coarer, E., Marcelin, M., et al. 1996, A\&A 315,243

Serlemitsos, P. J., Jalota, L., Soong, Y., et al. 1995, PASJ, 47, 105

Smith, R. C. 1997, AJ, 114, 2664

Sollerman, J., Ghavamian, P., Lundqvist, P., Smith, R. C., et al. 2003, A\&A, 407, 249

Tanaka, Y., Inoue, H., \& Holt, S. S. 1994, PASJ, 46, L37

Tanaka, T., Allafort, A., Ballet, J., et al. 2011, ApJ, 740, 51

Ueno, M., Sato, R., Kataoka, J., et al. 2007, PASJ, 59, 171

Valinia, A., \& Marshall, F. E. 1998, ApJ, 505, 134

Vink, J., Kaastra, J. S., \& Bleeker, J. A. M. 1997, A\&A, 328, 628

Vink, J., Bleeker, J., van der Heyden, K., et al. 2006, ApJ, 648, L33

Vink, J., Yamazaki, R., Helder, E. A., \& Schure, K. M. 2010, ApJ, 722, 1727

Völk, H. J., Berezhko, E. G., Ksenofontov, L. T. 2005, A\&A, 433, 229

Williams, B. J., Blair, W. P., Blondin, J. M., et al. 2011, ApJ, 741, 96

Whiteoak, J. B. Z., \& Green, A. J. 1996, A\&AS, 118, 329

Yamaguchi, H., Koyama, K., Nakajima, H., et al. 2008, PASJ, 60, 123

Yamaguchi, H., Koyama, K., \& Uchida, H. 2011, PASJ, 63, 837

Yuan, Q., Liu, S., Fan, Z., Bi, X., \& Fryer, C. L. 2011, ApJ, 735, 120

Zirakashvili, V. N., \& Aharonian, F. A. 2007, A\&A, 465, 695

Zirakashvili, V. N., \& Aharonian, F. A. 2010, ApJ, 708, 965 